\title{
Passive Localization Using Time Difference of Arrival and Frequency Difference of Arrival
}

\author{
Xiansheng Guo ${ }^{1,2}$, Yan Zhang3, Botao Zeng1 \\ ${ }^{1}$ Department of EE, University of Electronic Science and Technology of China, Chengdu, China \\ ${ }^{2}$ Wuhu Overseas Students Pioneer Park, Wuhu, China \\ ${ }^{3}$ Chengdu University, Chengdu, China \\ Email:xsguo@uestc.edu.cn
}

How to cite this paper: Guo, X.S., Zhang, Y. and Zeng, B.T. (2018) Passive Localization Using Time Difference of Arrival and Frequency Difference of Arrival. Journal of Computer and Communications, 6, 65-73. https://doi.org/10.4236/jcc.2018.61007

Received: September 20, 2017 Accepted: December 26, 2017 Published: December 29, 2017

\begin{abstract}
In order to improve the accuracy and engineering feasibility of four-Satellite localization system, the frequency difference measurement is introduced to the four-Satellite TDOA (Time Difference of Arrival) localization algorithm. The TDOA/FDOA (Frequency Difference of Arrival) localization algorithm is used to optimize the GDOP (geometric dilution of precision) of four-Satellite localization. The simulation results show that the absolute position measurement accuracy has little influence on TDOA/FDOA localization accuracy as compared with TDOA localization. Under the same conditions, TDOA/FDOA localization has better accuracy and its GDOP shows more uniform distribution in diamond configuration case. The localization accuracy of four-Satellite TDOA/FDOA is better than the localization accuracy of four-Satellite TDOA.
\end{abstract}

\section{Keywords}

Four-Satellite Localization, TDOA (Time Difference of Arrival), FDOA (Frequency Difference of Arrival),

GDOP (Geometric Dilution of Precision), Passive Localization

\section{Introduction}

With the development of space technology, high precision passive localization based on small satellite platform plays an important role in civil and military fields and has broad application prospects [1]. The development trend of space electronic reconnaissance technology is to increase the measurement information of target and adopt joint positioning method to improve positioning accuracy and increase reconnaissance range [2]. In recent years, the two-satellite TDOA/FDOA localization method has been developed both in domestic and 
global position fields, which lays a foundation for the research of TDOA/FDOA localization system [3] [4] [5]. There are also literatures of multi-statellite TDOA/FDOA localization method [6] [7] [8] [9] [10], However, they seldom considered the four-satellite TDOA/FDOA joint localization problem. In this paper, the localization performance of the four-satellite TDOA/FDOA localization method is studied. Compared with the TDOA localization system, the advantages of the diamond configuration and accuracy of the absolute position measurement are emphasized.

\section{Principle of Four-Satellite TDOA}

Suppose that the model of the three-dimensional TDOA localization system (as shown in Figure 1) consists of 1 main satellite and three subsatellites. $S_{0}$ is the main satellite, $S_{1}, S_{2}, S_{3}$ are the subsatellites. Their coordinates are denoted as $S_{i}\left(x_{i}, y_{i}, z_{i}\right)$, where $i=0,1,2,3 . \boldsymbol{p}(x, y, z)$ is the location of the target radiation source to be estimated.

According to the position of each satellite, the distance differences between the main satellite and the other three subsatellites satisfy the following equations:

$$
\left\{\begin{array}{l}
\Delta r_{i}=r_{i}-r_{0} \\
r_{0}=\sqrt{\left(x-x_{0}\right)^{2}+\left(y-y_{0}\right)^{2}+\left(z-z_{0}\right)^{2}} \\
r_{i}=\sqrt{\left(x-x_{i}\right)^{2}+\left(y-y_{i}\right)^{2}+\left(z-z_{i}\right)^{2}}
\end{array}\right.
$$

where $r_{i}$ is the radial distance between the satellite $i$ and the target where $i=0,1,2,3$. Equations (2) can be obtained with simplification and rearrangement of Equation (1):

$$
\left(x_{0}-x_{i}\right) x+\left(y_{0}-y_{i}\right) y+\left(z_{0}-z_{i}\right) z=r_{0} \Delta r_{i}+k_{i}
$$

where $k_{i}=\frac{1}{2}\left[\Delta r_{i}^{2}+\left(x_{0}^{2}+y_{0}{ }^{2}+z_{0}{ }^{2}\right)-\left(x_{i}^{2}+y_{i}^{2}+z_{i}^{2}\right)\right]$. The matrix representation of Equation (2) can be given by

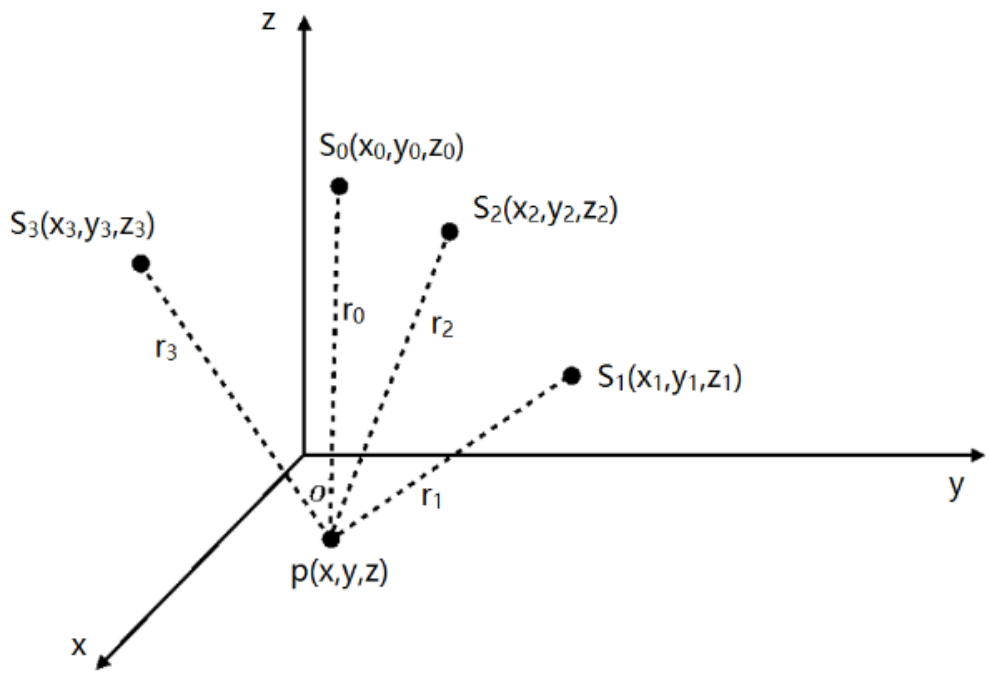

Figure 1. Principle of four-satellite TDOA. 


$$
A x=f
$$

where

$$
\begin{gathered}
\boldsymbol{A}=\left[\begin{array}{l}
x_{0}-x_{1}, y_{0}-y_{1}, z_{0}-z_{1} \\
x_{0}-x_{2}, y_{0}-y_{2}, z_{0}-z_{2} \\
x_{0}-x_{3}, y_{0}-y_{3}, z_{0}-z_{3}
\end{array}\right], \\
\boldsymbol{x}=[x, y, z]^{T} \text {, and } \boldsymbol{f}=\left[r_{0} \Delta r_{1}+k_{1}, r_{0} \Delta r_{2}+k_{2}, r_{0} \Delta r_{3}+k_{3}\right]^{T} .
\end{gathered}
$$

Ideally for satellites location, $\operatorname{rank}(\boldsymbol{A})=3$, Equation (4) can be obtained by using pseudo inverse to solve Equation (3):

$$
\boldsymbol{x}=\left(\boldsymbol{A}^{\mathrm{T}} \boldsymbol{A}\right)^{-1} \boldsymbol{A}^{\mathrm{T}} \boldsymbol{f}
$$

The accuracy analysis of four-satellite TDOA localization algorithm is as follows.

Differentiating $\Delta r_{i}$ :

$$
\begin{aligned}
\mathrm{d}\left(\Delta r_{i}\right)= & \left(c_{i x}-c_{0 x}\right) \mathrm{d} x+\left(c_{i y}-c_{0 y}\right) \mathrm{d} y+\left(c_{i z}-c_{0 z}\right) \mathrm{d} z \\
& -c_{i x} \mathrm{~d} x_{i}-c_{i y} \mathrm{~d} y_{i}-c_{i z} \mathrm{~d} z_{i}+c_{0 x} \mathrm{~d} x_{0}+c_{o y} \mathrm{~d} y_{0}+c_{0 z} \mathrm{~d} z_{0}
\end{aligned}
$$

where $c_{i x}=\frac{x-x_{i}}{r_{i}}, c_{i y}=\frac{y-y_{i}}{r_{i}}, c_{i z}=\frac{z-z_{i}}{r_{i}}$.

Let

$$
\begin{gathered}
\boldsymbol{C}=\left[\begin{array}{l}
c_{1 x}-c_{0 x}, c_{1 y}-c_{0 y}, c_{1 z}-c_{0 z} \\
c_{2 x}-c_{0 x}, c_{2 y}-c_{0 y}, c_{2 z}-c_{0 z} \\
c_{3 x}-c_{0 x}, c_{3 y}-c_{0 y}, c_{3 z}-c_{0 z}
\end{array}\right], \mathrm{d} \boldsymbol{X}=[\mathrm{d} x, \mathrm{~d} y, \mathrm{~d} z]^{T}, \\
\mathrm{~d} \boldsymbol{Y}=\left[\mathrm{d} \Delta r_{1}, \mathrm{~d} \Delta r_{2}, \mathrm{~d} \Delta r_{3}\right]^{T}, \mathrm{~d} \boldsymbol{S}_{i}=\left[\begin{array}{ll}
\mathrm{d} x_{i}, \mathrm{~d} y_{i}, \mathrm{~d} z_{i}
\end{array}\right]^{T}, \boldsymbol{U}_{0}=\left[\begin{array}{l}
c_{0 x}, c_{0 y}, c_{0 z} \\
c_{0 x}, c_{0 y}, c_{0 z} \\
c_{0 x}, c_{0 y}, c_{0 z}
\end{array}\right], \\
\boldsymbol{U}_{1}=\left[\begin{array}{ccc}
c_{1 x} & c_{1 y} & c_{1 z} \\
0 & 0 & 0 \\
0 & 0 & 0
\end{array}\right], \boldsymbol{U}_{2}=\left[\begin{array}{ccc}
0 & 0 & 0 \\
c_{2 x} & c_{2 y} & c_{2 z} \\
0 & 0 & 0
\end{array}\right], \boldsymbol{U}_{3}=\left[\begin{array}{ccc}
0 & 0 & 0 \\
0 & 0 & 0 \\
c_{3 x} & c_{3 y} & c_{3 z}
\end{array}\right] .
\end{gathered}
$$

We can obtain

$$
\boldsymbol{C} \cdot \mathrm{d} \boldsymbol{X}=\mathrm{d} \boldsymbol{Y}-\boldsymbol{U}_{0} \mathrm{~d} \boldsymbol{S}_{0}+\boldsymbol{U}_{1} \mathrm{~d} \boldsymbol{S}_{1}+\boldsymbol{U}_{2} \mathrm{~d} \boldsymbol{S}_{2}+\boldsymbol{U}_{3} \mathrm{~d} \boldsymbol{S}_{3}
$$

Let $\boldsymbol{B}=\left(\boldsymbol{C}^{\mathrm{T}} \boldsymbol{C}\right)^{-1} \boldsymbol{C}^{\mathrm{T}}$. Since the main satellite has an error in measuring the arrival time of the radiation source signal, and the error exists in each time difference measurement. The measurement errors of each $\Delta r_{i}$ are related. we assumed that the mean of the measurement error is 0 after the systematic calibration. Moreover, each element of satellite position error is uncorrelated and each satellite position is uncorrelated. The covariance matrix of the target radiation source localization error can be obtained as follows.

$$
\begin{aligned}
\boldsymbol{P}_{\mathrm{d} \hat{\boldsymbol{X}}}= & \mathrm{E}[\mathrm{d} \hat{\boldsymbol{X}} \mathrm{d} \hat{\boldsymbol{X}}]=\boldsymbol{B}\left\{\mathrm{E}\left[\mathrm{d} \boldsymbol{Y} \mathrm{d} \boldsymbol{Y}^{\mathrm{T}}\right]\right\}+\boldsymbol{U}_{0} \mathrm{E}\left[\mathrm{d} \boldsymbol{S}_{0} \mathrm{~d} \boldsymbol{S}_{0}{ }^{\mathrm{T}}\right] \boldsymbol{U}_{0}{ }^{\mathrm{T}} \\
& +\boldsymbol{U}_{1} \mathrm{E}\left[\mathrm{d} \boldsymbol{S}_{1} \mathrm{~d} \boldsymbol{S}_{1}^{\mathrm{T}}\right] \boldsymbol{U}_{1}^{\mathrm{T}}+\boldsymbol{U}_{2} \mathrm{E}\left[\mathrm{d} \boldsymbol{S}_{2} \mathrm{~d} \boldsymbol{S}_{2}{ }^{\mathrm{T}}\right] \boldsymbol{U}_{2}{ }^{\mathrm{T}}+\boldsymbol{U}_{3} \mathrm{E}\left[\mathrm{d} \boldsymbol{S}_{3} \mathrm{~d} \boldsymbol{S}_{3}{ }^{\mathrm{T}}\right] \boldsymbol{U}_{3}{ }^{\mathrm{T}}
\end{aligned}
$$


where $\mathrm{E}\left[\mathrm{d} \boldsymbol{Y} d \boldsymbol{Y}^{\mathrm{T}}\right]=\left[\begin{array}{ccc}\sigma_{\Delta r_{1}}^{2} & \eta_{12} \sigma_{\Delta r_{1}} \sigma_{\Delta r_{2}} & \eta_{12} \sigma_{\Delta r_{1}} \sigma_{\Delta r_{3}} \\ \eta_{12} \sigma_{\Delta r_{1}} \sigma_{\Delta r_{2}} & \sigma_{\Delta r_{2}}^{2} & \eta_{23} \sigma_{\Delta r_{2}} \sigma_{\Delta r_{3}} \\ \eta_{13} \sigma_{\Delta r_{1}} \sigma_{\Delta r_{3}} & \eta_{23} \sigma_{\Delta r_{2}} \sigma_{\Delta r_{3}} & \sigma_{\Delta r_{3}}^{2}\end{array}\right], \sigma_{\Delta r_{i}}^{2}$ is the variance of the measurement error of the distance between the satellite $i$ and the main satellite. $\eta_{i j}$ is the correlation coefficient between $\Delta r_{i}$ and $\Delta r_{j}$. We assume that the variance of satellite position error on each component is the same, that is $\sigma_{x_{i}}^{2}=\sigma_{y_{i}}^{2}=\sigma_{z_{i}}^{2}$. We can obtain the covariance matrix of the satellite position error as Equation (8).

$$
\mathrm{E}\left[\mathrm{d} \boldsymbol{S}_{i} \mathrm{~d} \boldsymbol{S}_{i}^{\mathrm{T}}\right]=\mathrm{E}\left[\left[\mathrm{d} x_{i}, \mathrm{~d} y_{i}, \mathrm{~d} z_{i}\right]^{T}\left[\mathrm{~d} x_{i}, \mathrm{~d} y_{i}, \mathrm{~d} z_{i}\right]\right]=\operatorname{diag}\left(\left[\sigma_{x_{i}}^{2}, \sigma_{y_{i}}^{2}, \sigma_{z_{i}}^{2}\right]\right)
$$

The covariance matrix of the target radiation source position error is as Equation (9).

$$
\mathrm{E}\left[\mathrm{d} \boldsymbol{X} \mathrm{d} \boldsymbol{X}^{\mathrm{T}}\right]=\left[[\mathrm{d} x, \mathrm{~d} y, \mathrm{~d} z]^{T}[\mathrm{~d} x, \mathrm{~d} y, \mathrm{~d} z]^{T}\right]=\operatorname{diag}\left(\left[\sigma_{x}^{2}, \sigma_{y}^{2}, \sigma_{z}^{2}\right]\right)
$$

According to the definition of the GDOP, the GDOP of TDOA localization in three dimensional space can be calculated as Equation (10).

$$
\text { GDOP }=\sqrt{\operatorname{trace}\left(\mathrm{E}\left[\mathrm{d} \boldsymbol{X} \mathrm{d} \boldsymbol{X}^{\mathrm{T}}\right]\right)}
$$

\section{Principle of Four-Satellite TDOA/FDOA}

On the basis of the four-satellite TDOA localization algorithm, the four-satellite TDOA/FDOA localization algorithm adds frequency difference information. The coordinate of the target is recorded as $\boldsymbol{p}(x, y, z)$. The coordinate of the main satellite is recorded as $S_{0}\left(x_{0}, y_{0}, z_{0}\right)$. The speed of the main satellite is recorded as $\boldsymbol{v}_{0}\left(v_{x 0}, v_{y 0}, v_{z 0}\right)$. The coordinate of the subsatellites is recorded as $S_{i}\left(x_{i}, y_{i}, z_{i}\right)$. The speed of the subsatellite is recorded as $v_{i}\left(v_{x i}, v_{y i}, v_{z i}\right)$, where $i=1,2,3$. According to the time difference and frequency difference of the target radiation source arriving at the main satellite and subsatellites, we can obtain that

$$
\begin{aligned}
f_{i}=\mathrm{c} \cdot \text { tdoa }_{i}= & \sqrt{\left(x-x_{0}\right)^{2}+\left(y-y_{0}\right)^{2}+\left(z-z_{0}\right)^{2}} \\
& -\sqrt{\left(x-x_{i}\right)^{2}+\left(y-y_{i}\right)^{2}+\left(z-z_{i}\right)^{2}} \\
f_{j}=\mathrm{c} \cdot \frac{\text { fdoa }_{i}}{f}= & \frac{v_{x_{0}}\left(x-x_{0}\right)+v_{y_{0}}\left(y-y_{0}\right)+v_{z_{0}}\left(z-z_{0}\right)}{\sqrt{\left(x-x_{0}\right)^{2}+\left(y-y_{0}\right)^{2}+\left(z-z_{0}\right)^{2}}} \\
& -\frac{v_{x_{i}}\left(x-x_{i}\right)+v_{y_{i}}\left(y-y_{i}\right)+v_{z_{i}}\left(z-z_{i}\right)}{\sqrt{\left(x-x_{i}\right)^{2}+\left(y-y_{i}\right)^{2}+\left(z-z_{i}\right)^{2}}}
\end{aligned}
$$

where $i=1,2,3, j=4,5,6$. And $t d o a_{i}$ is the time difference of arrival, fdoa $_{i}$ is the frequency difference of arrival, $f$ is the radiation frequency of radiation source, c is the light speed. By solving the least square solution of the equation, the target position can be obtained. The purpose of this paper is to analyze the accuracy of the four-Satellite localization, so the solution process of the equation is no longer exhausted. 
Using first order linear approximation expansion for $t d o a_{i}, f_{d o a_{i}},(x, y, z)$, $\left(x_{i}, y_{i}, z_{i}\right)$ and $v_{i}\left(v_{x i}, v_{y i}, v_{z i}\right)$, we can obtain the Equation (13).

$$
\begin{aligned}
& c[\text { TDOA, FDOA } / f]^{T} \\
& =\boldsymbol{B}[\mathrm{d} x, \mathrm{~d} y, \mathrm{~d} z]^{T}+\sum_{i=0}^{3} \boldsymbol{B}_{1 i}\left[\mathrm{~d} x_{i}, \mathrm{~d} y_{i}, \mathrm{~d} z_{i}\right]^{T}+\sum_{i=0}^{3} \boldsymbol{B}_{2 i}\left[\mathrm{~d} v_{x i}, \mathrm{~d} v_{y i}, \mathrm{~d} v_{z i}\right]^{T}
\end{aligned}
$$

where

$$
\begin{gathered}
\text { TDOA }=\left[\text { tdoa }_{1}, t \text { toa }_{2}, t \text { toa }_{3}\right]^{T}, \quad \boldsymbol{F D O A}=\left[f \text { doa }_{1}, f d o a_{2}, f d o a_{3}\right]^{T} \\
\boldsymbol{B}=\left[\begin{array}{lll}
\partial f_{1} / \partial x & \partial f_{1} / \partial y & \partial f_{1} / \partial z \\
\partial f_{2} / \partial x & \partial f_{2} / \partial y & \partial f_{2} / \partial z \\
\partial f_{3} / \partial x & \partial f_{3} / \partial y & \partial f_{3} / \partial z \\
\partial f_{4} / \partial x & \partial f_{4} / \partial y & \partial f_{4} / \partial z \\
\partial f_{5} / \partial x & \partial f_{5} / \partial y & \partial f_{5} / \partial z \\
\partial f_{6} / \partial x & \partial f_{6} / \partial y & \partial f_{6} / \partial z
\end{array}\right], \quad \boldsymbol{B}_{1 i}=\left[\begin{array}{lll}
\partial f_{1} / \partial x_{i} & \partial f_{1} / \partial y_{i} & \partial f_{1} / \partial z_{i} \\
\partial f_{2} / \partial x_{i} & \partial f_{2} / \partial y_{i} & \partial f_{2} / \partial z_{i} \\
\partial f_{3} / \partial x_{i} & \partial f_{3} / \partial y_{i} & \partial f_{3} / \partial z_{i} \\
\partial f_{4} / \partial x_{i} & \partial f_{4} / \partial y_{i} & \partial f_{4} / \partial z_{i} \\
\partial f_{5} / \partial x_{i} & \partial f_{5} / \partial y_{i} & \partial f_{5} / \partial z_{i} \\
\partial f_{6} / \partial x_{i} & \partial f_{6} / \partial y_{i} & \partial f_{6} / \partial z_{i}
\end{array}\right]
\end{gathered}
$$

Take the upper equation as

$$
\begin{aligned}
\boldsymbol{B}[\mathrm{d} x, \mathrm{~d} y, \mathrm{~d} z]^{T}= & c[\boldsymbol{T D O} \boldsymbol{A}, \boldsymbol{F D O A} / f]^{T}-\sum_{i=0}^{3} \boldsymbol{B}_{1 i}\left[\mathrm{~d} x_{i}, \mathrm{~d} y_{i}, \mathrm{~d} z_{i}\right]^{T} \\
& -\sum_{i=0}^{3} \boldsymbol{B}_{2 i}\left[\mathrm{~d} v_{x i}, \mathrm{~d} v_{y i}, \mathrm{~d} v_{z i}\right]^{T}
\end{aligned}
$$

Divide the measurement accuracy of the subsatellite position into measurement accuracy of absolute position and measurement accuracy of relative position. That is

$$
\left[\mathrm{d} x_{i}, \mathrm{~d} y_{i}, \mathrm{~d} z_{i}\right]^{T}=\left[\mathrm{d} x_{0}, \mathrm{~d} y_{0}, \mathrm{~d} z_{0}\right]^{T}+\left[\mathrm{d} x_{i}^{\prime}, \mathrm{d} y_{i}^{\prime}, \mathrm{d} z_{i}^{\prime}\right]^{T}
$$

The right $\left[\mathrm{d} x_{i}^{\prime}, \mathrm{d} y_{i}^{\prime}, \mathrm{d} z_{i}^{\prime}\right]^{T}$ is the measurement accuracy of relative position between main satellite and subsatellites. $\left[\mathrm{d} x_{0}, \mathrm{~d} y_{0}, \mathrm{~d} z_{0}\right]^{T}$ is the measurement accuracy of absolute position of main satellite. We can obtain the Equation (15).

$$
\begin{aligned}
\boldsymbol{B}[\mathrm{d} x, \mathrm{~d} y, \mathrm{~d} y]^{T}= & c[\text { TDOA,FDOA } / f]^{T}-\sum_{i=0}^{3} \boldsymbol{B}_{1 i}\left[\mathrm{~d} x_{0}, \mathrm{~d} y_{0}, \mathrm{~d} y_{0}\right]^{T} \\
& -\sum_{i=1}^{3} \boldsymbol{B}_{1 i}\left[\mathrm{~d} x_{i}^{\prime}, \mathrm{d} y_{i}^{\prime}, \mathrm{d} z_{i}^{\prime}\right]^{T}-\sum_{i=0}^{3} \boldsymbol{B}_{2 i}\left[\mathrm{~d} v_{x i}, \mathrm{~d} v_{y i}, \mathrm{~d} v_{z i}\right]^{T}
\end{aligned}
$$

Let $\quad \boldsymbol{K}_{1}=\sum_{i=0}^{3} \boldsymbol{B}_{1 i}, \quad \boldsymbol{K}_{2}=\boldsymbol{B}_{11}, \quad \boldsymbol{K}_{3}=\boldsymbol{B}_{12}, \quad \boldsymbol{K}_{4}=\boldsymbol{B}_{13}, \quad \boldsymbol{K}_{5}=\boldsymbol{B}_{20}, \quad \boldsymbol{K}_{6}=\boldsymbol{B}_{21}$, $\boldsymbol{K}_{7}=\boldsymbol{B}_{22}, \quad \boldsymbol{K}_{8}^{j=0}=\boldsymbol{B}_{23}$. And we assume that the measurement accuracy of position, the measurement accuracy of speed, the measurement accuracy of TDOA, the measurement accuracy of FDOA are uncorrelated. We can obtain Equation (16) by matrix operation.

$$
\begin{aligned}
& \mathrm{E}\left[[\mathrm{d} x, \mathrm{~d} y, \mathrm{~d} z]^{\mathrm{T}}[\mathrm{d} x, \mathrm{~d} y, \mathrm{~d} z]\right] \\
& =c^{2} \boldsymbol{B}^{-1} \boldsymbol{M}\left(\boldsymbol{B}^{-1}\right)^{\mathrm{T}}+\sum_{i=1}^{8} \boldsymbol{B}^{-1} \boldsymbol{K}_{i} \boldsymbol{E}_{i} \boldsymbol{K}_{i}^{\mathrm{T}}\left(\boldsymbol{B}^{-1}\right)^{\mathrm{T}}
\end{aligned}
$$

where $\boldsymbol{M}$ is the correlation matrix of measurement accuracy between TDOA and FDOA. $\quad \boldsymbol{E}_{i}$ is the correlation matrix of the measurement accuracy of position and the measurement accuracy of speed. 
The positioning accuracy can be expressed by the measuring accuracy of 3 orthogonal directions as Equation (17)

$$
G D O P=\sqrt{\sigma_{x}^{2}+\sigma_{y}^{2}+\sigma_{z}^{2}}
$$

From the above analysis results, we can see that the positioning accuracy is calculated by $\boldsymbol{M}$ and $\boldsymbol{E}_{i}$.

We assume that the mean of the TDOA signal and the FDOA signal measurement accuracy is 0 , and they are uncorrelated. We can obtain that

$$
\boldsymbol{M}=\operatorname{diag}\left(\left[\sigma_{t}^{2} \boldsymbol{I}_{3}, \frac{\sigma_{f}^{2}}{f^{2}} \boldsymbol{I}_{3}\right]\right)
$$

where $I_{3}$ is a $3 \times 3$ unit matrix, $\sigma_{t}$ is the measurement accuracy of time difference, $\sigma_{f}$ is the measurement accuracy of frequency difference.

The absolute position measurement accuracy component $\mathrm{d} x_{0}, \mathrm{~d} y_{0}, \mathrm{~d} z_{0}$ are considered to be independent of the random variables. Therefore, the correlation matrix of four-satellite absolute position measurement accuracy is

$$
\boldsymbol{E}_{1}=\frac{\sigma_{a s}^{2}}{3} \boldsymbol{I}_{3}
$$

where $\sigma_{a s}$ is the measurement accuracy of absolute position.

Comprehensively considering the measurement accuracy of relative position and the ranging accuracy, the correlation matrix of relative position measurement accuracy can be represented as

$$
\boldsymbol{E}_{i}=\operatorname{diag}\left(\left[\frac{\sigma_{b s}^{2}-\sigma_{d}^{2}}{2}, \frac{\sigma_{b s}^{2}-\sigma_{d}^{2}}{2}, \sigma_{d}^{2}\right]\right)
$$

where $i=2,3,4$, and $\sigma_{b s}$ is the measurement accuracy of relative position, $\sigma_{d}$ is the ranging accuracy.

The correlation matrix of speed measurement accuracy can be represented as $\boldsymbol{E}_{j}=\sigma_{v}^{2} \boldsymbol{I}_{3}$ where $\sigma_{v}$ is measurement accuracy of speed.

\section{Simulation Results}

We assume that the satellite orbit altitude is $200 \mathrm{~km}$ and the length of baseline is $60 \mathrm{~km}$. The $\mathrm{Y}$ configuration, $\mathrm{T}$ configuration and diamond configuration are adopted respectively. The measurement accuracy of time difference is $50 \mathrm{~ns}$. The measurement accuracy of frequency difference is $10 \mathrm{~Hz}$. The measurement accuracy of speed is $1 \mathrm{~m} / \mathrm{s}$. The absolute position measurement accuracy is $150 \mathrm{~m}$. The relative position measurement accuracy is $50 \mathrm{~m}$. The GDOP distributions of TDOA localization and TDOA/FDOA localization are obtained by simulation, as shown in Figures 2-4.

Analysis and comparison of Figures 2-4 show that under the same parameters, the accuracy of TDOA/FDOA localization is better than that of TDOA localization. And for the diamond configuration, the improvement of TDOA/FDOA localization accuracy is especially obvious. The GDOP of TDOA/FDOA localization regards subastral point as center and uniformly distributed. But the TDOA localization of GDOP is unevenly distributed. 

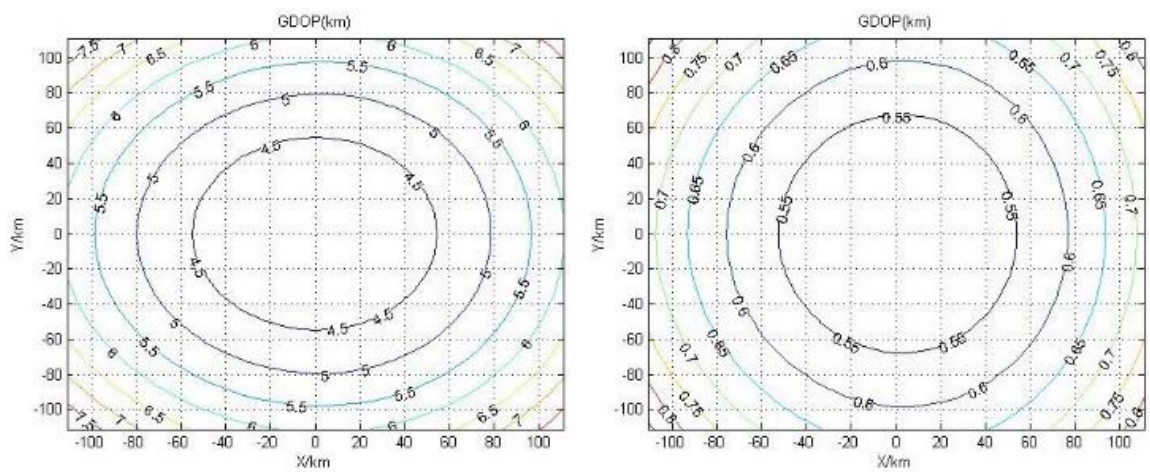

Figure 2. Y configuration, the GDOP distributions of TDOA localization (left) and TDOA/FDOA localization (right).
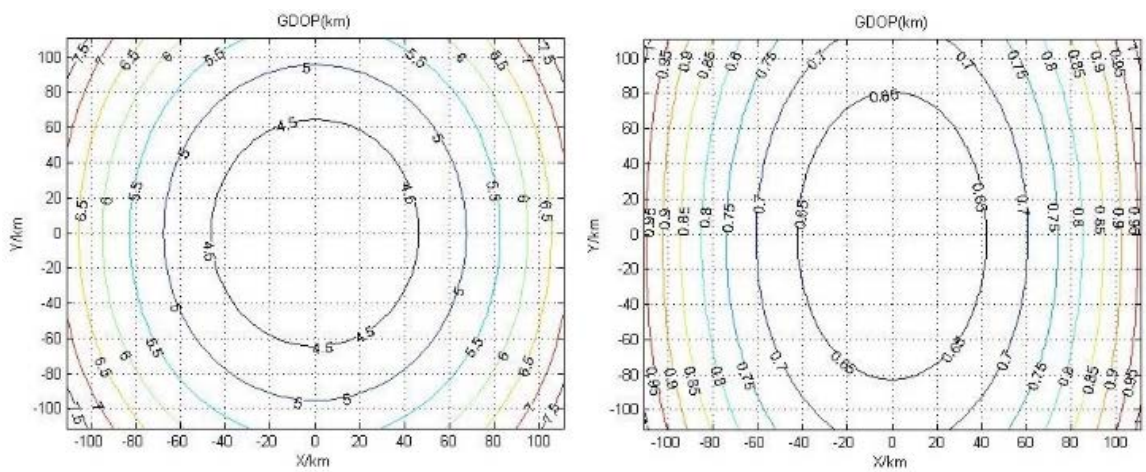

Figure 3. T configuration, the GDOP distributions of TDOA localization (left) and TDOA/FDOA localization (right).
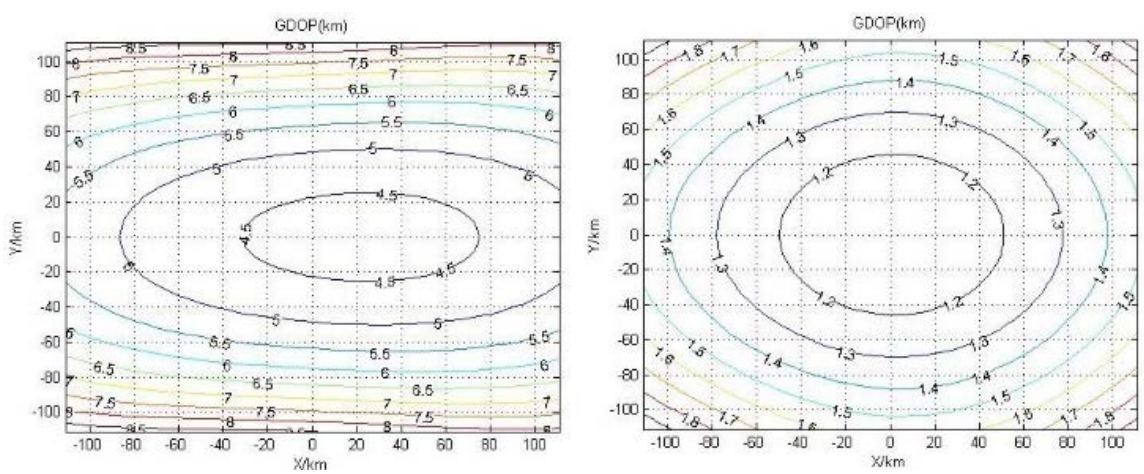

Figure 4. Diamond configuration, the GDOP distributions of TDOA localization (left) and TDOA/FDOA localization (right).

Let measurement accuracy of absolute position in continuous $0-300 \mathrm{~m}$ and continuous value. Select the $\mathrm{Y}$ configuration, and the remaining parameters remain unchanged. Taking a point on the $100 \mathrm{~km}$ circumference of the subastral point, the relationship of TDOA localization accuracy and TDOA/FDOA localization accuracy with the absolute position measurement accuracy are obtained respectively, as shown in Figure 5 and Figure 6.

Comparing Figure 5 and Figure 6, the localization accuracy of the two algorithms will be improved with the increase of absolute position measurement 


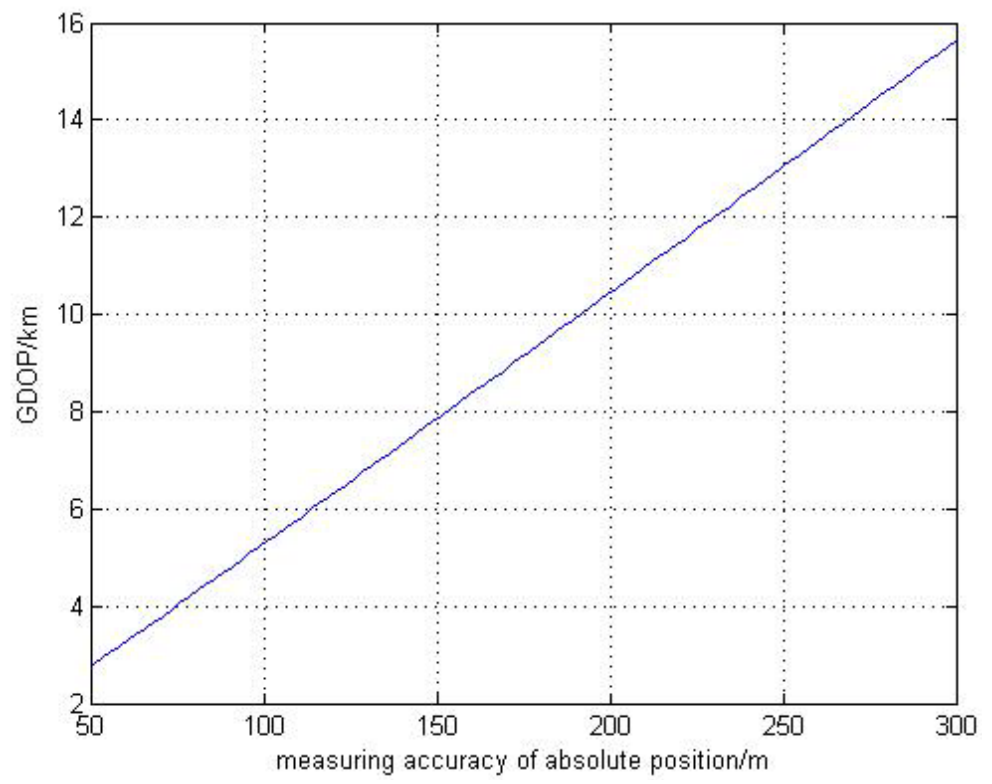

Figure 5. Relationship of TDOA localization accuracy with the absolute position measurement accuracy.

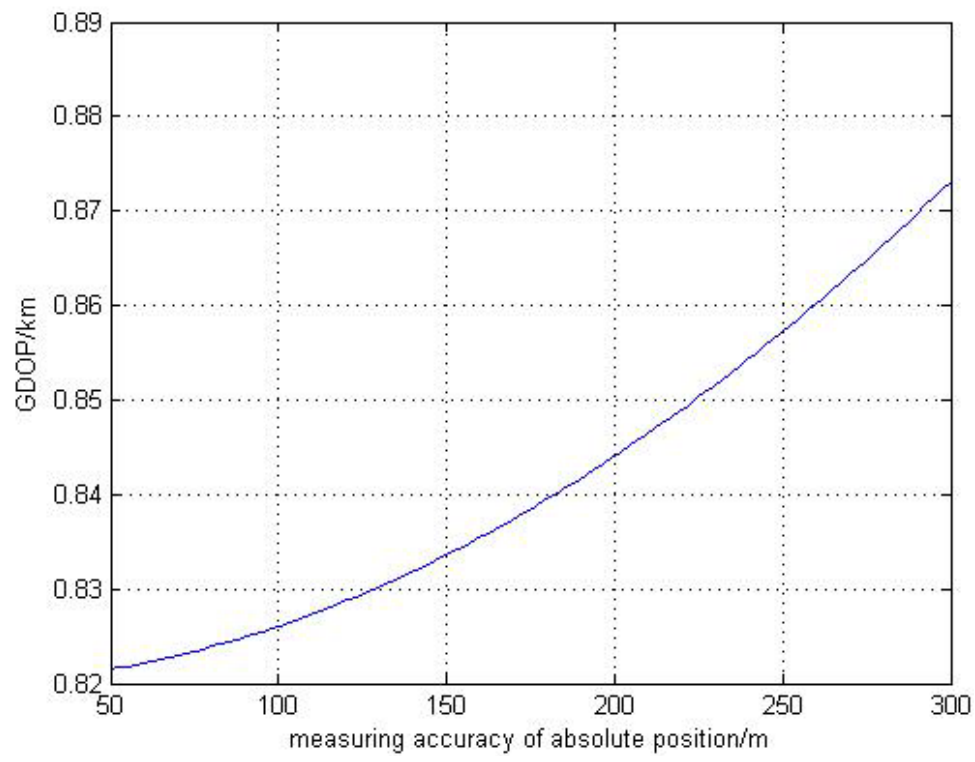

Figure 6. Relationship of TDOA/FDOA localization accuracy with the absolute position measurement accuracy.

accuracy. But the localization accuracy of TDOA is greatly affected by absolute position measurement accuracy. The localization accuracy of TDOA/FDOA is affected by absolute position measurement accuracy very little. Compared with TDOA localization, the effect is almost negligible.

\section{Conclusion}

The principles of four-satellite TDOA and TDOA/FDOA localization algorithms are analyzed, and the GDOP expressions of the two algorithms are derived re- 
spectively. Through simulation analysis, some useful conclusions can be drawn as follows.

With the same parameters, TDOA/FDOA localization algorithm shows better accuracy than TDOA localization algorithm.

When using the diamond configuration, as compared with the TDOA localization algorithm, the TDOA/FDOA localization algorithm not only has better localization accuracy, but also improves the GDOP distribution uniformity. It solves the problem of uneven distribution of diamond configuration and increases the engineering feasibility.

As compared with the TDOA localization, the absolute position measurement accuracy of satellite has almost no effect on the localization accuracy of TDOA/ FDOA, which reduces the localization error caused by unavoidable absolute position measuring factors.

\section{Acknowledgements}

This work is supported by the National Natural Science Foundation of China under Grant (No. 61201277, No. 61371184, No. 61671137, No. 61771114) and the Fundamental Research Funds for the Central Universities (No. ZYGX2016J028).

\section{References}

[1] Hu, L.Z. (2004) The Summarization of Passive Locating Technique. Electronic Warfare.

[2] Wu, J. (2009) Current Status and Development of Foreign Space Reconnaissance Systems. Telecommunication Engineering, 49, 108-114.

[3] Chang, X.Y., Xie, B., Zhang, X.M. and Lan, D. (2013) Target Location Accuracy Analysis of Dual-satellites Location System Using TDOA and FDOA. Spacecraft Engineering, 22, 35-42.

[4] Guo, F.C. and Fan, Y. (2006) Analysis of Dual Satellites Geo Location Method Using TDOA and FDOA. Aerospace Eletronic Warfare, 22, 20-23.

[5] Zhang, Y., Sheng, W.D. and Guo, F.C. (2007) Low Orbit Dual-Satellites Passive Location Algorithm and Its Precision Analysis. Journal of Chinese Inertial Technology, 15, 188-192.

[6] Liu, D.J. (2012) Study of Joint Passive Location Using Time Difference of Arrival and Frequency Difference of Arrival Measurements. Xidian University.

[7] Ulman, R.J. and Gerantiotis, E. (2001) Motion Detection Using TDOA and FDOA Measurements. IEEE Transactions on Aerospace \& Electronic Systems, 37, 759-764. https://doi.org/10.1109/7.937490

[8] Pan, Q.G., Yan, M. and Liao, G.S. (2005) Joint Passive Location Using TDOA and FDOA in Multi Stations. Radar Science and Technology, 3, 332-335.

[9] Yin, J.R., Cheng, F.D. and Li, S. (2008) Joint Passive Locating Using TDOA and Doppler Shift Based on Multi-Stations. Modern Radar, 30, 61-64.

[10] Guo, X., Chu, L. and Sun, X. (2016) Accurate Localization of Multiple Sources Using Semidefinite Programming Based on Incomplete Range Matrix. IEEE Sensors Journal, 16, 5319-5324. https://doi.org/10.1109/JSEN.2016.2558184 